\title{
The Cancer Genome Atlas
}

\author{
B. Minu Chandra Muddabhaktuni ${ }^{1}$ Venkata Pradeep Babu Koyyala ${ }^{1}$
}

${ }^{1}$ Department of Medical Oncology, Rajiv Gandhi Cancer Institute and Research Centre, Ahmedabad, Gujarat, India

Ind J Med Paediatr Oncol 2021;42:353-355.

\section{What Is the Cancer Genome Atlas-Origin?}

The Cancer Genome Atlas (TCGA) is a landmark cancer genomics program started in 2006 to molecularly characterize different types of cancers. This was a joint effort of the US government-funded National Cancer Institute and National Human Genome Research Institute under the guidance of Dr. Jean Claude Zenklusen. ${ }^{1}$

\section{Goal of the Cancer Genome Atlas?}

The goal was to illustrate that advanced genomic data could be utilized by the scientific community to generate statistically significant and biologically sound conclusions.

Over the next dozen years, it was able to generate 2.5 petabytes $\left(1,000,000,000,000,000\right.$ or $10^{15}$ bytes $)$ of genomic, epigenomic (epigenetic), transcriptomic, and proteomic data.

\section{Methods}

Tissue accrual was the first and the most challenging step. Requirement of the tissue was different for each cancer and tissue type. Each tissue type for a specific cancer had certain "standards" for accrual. For example, in case of brain tumors, the sample should not contain necrosis of more than $50 \%$ and tumor nuclei should consist of more than $80 \%$ in the viable portion of the tumor with the total tumor sample weighing a minimum of $200 \mathrm{mg}$. Every sample was always matched with a source that provided the germline DNA (e.g., blood or purified DNA).

In the beginning, TCGA started a pilot project to characterize three different types of tumors, namely glioblastoma multiforme, lung, and ovarian cancer. After the success of the pilot project, a phase II project was planned to characterize 20 to 25 different types of cancers. Finally, in
Address for correspondence B. Minu Chandra Muddabhaktuni, MBBS, MD, DM (medical oncology), Department of Medical Oncology, GCRI, Ahmedabad, Gujrat 380016, India (e-mail: minuchandra121@gmail.com).

December 2013, TCGA completed the sample collection with close to 20,000 biospecimen pairs. Each specimen was analyzed as per - Fig. 1. Information was grouped into relevant subsets. The target was to sequence 20 to 25 different types of cancers, but a total of 33 primary cancers including 11 rare varieties have been sequenced till date.

\section{The Cancer Genome Atlas Outcomes and Impact}

\section{The Pan-Cancer Atlas}

It is the culmination of more than a decade of hard work from TCGA. It provides a unique, deep, interconnected, far-reaching understanding of why, where, and how the human tumors arise. The visuals depicted represent the Pan-Cancer Atlas with a series of interconnected, shaded rings that form a collage to create a beautiful singular spectrum of ocular feast ( - Fig. 2). Like the research itself, the full impact of visualization is found in its cohesion. The visual contains three primary colors, representing the cell of origin patterns, signaling pathways, and oncogenic process. As we appreciate, the primary color slowly fades off and gives rise to a new color which took its birth from the previous. Similarly, the cell of origin patterns, signaling pathways, and oncogenic process might appear distinct, but they are deeply intertwined and indirectly give birth to the next.

\section{The Cell of Origin Patterns}

It emphasizes on the reclassification of the human tumors based on the molecular similarity, stating that the cell of origin influences but does not fully govern the classification.

\section{The Oncogenic Processes}

It gives a bird's-eye view of the oncogenic processes that lead to the development of human cancer. It carefully showcases
DOI https://doi.org/ $10.1055 / \mathrm{s}-0041-1735440$ ISSN 0971-5851
(C) 2021. Indian Society of Medical and Paediatric Oncology. This is an open access article published by Thieme under the terms of the Creative Commons Attribution-NonDerivative-NonCommercial-License, permitting copying and reproduction so long as the original work is given appropriate credit. Contents may not be used for commercial purposes, or adapted, remixed, transformed or built upon. (https://creativecommons.org/licenses/by-nc-nd/4.0/). Thieme Medical and Scientific Publishers Private Ltd. A-12, Second Floor, Sector -2, NOIDA -201301, India 


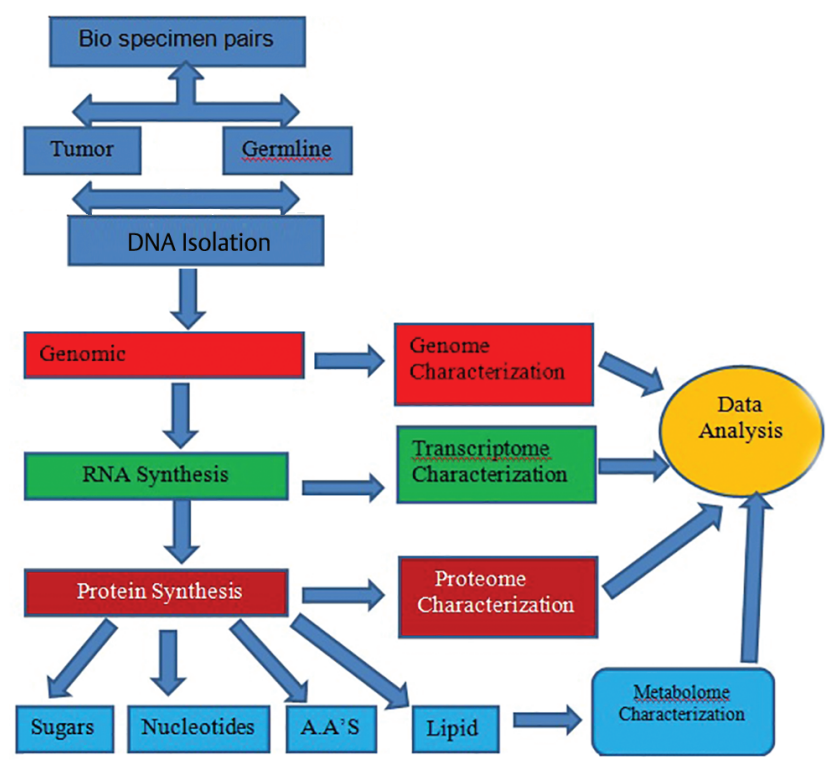

Fig. 1 Methodological overview of the Cancer Genome Atlas outcomes and impact.

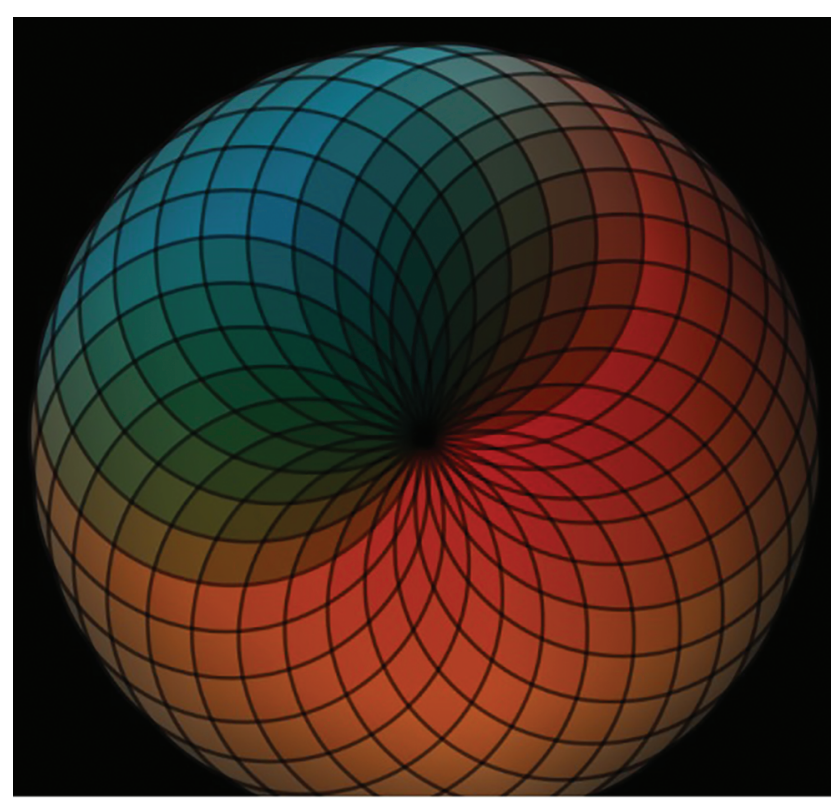

Fig. 2 Logo Pan-Cancer Atlas. Source-National Cancer Institute (the Cancer Genome Atlas).

the interplay between the somatic mutations and the germline genetic variants in the progression of cancer. It reinvents the governance of mutation on immune composition and cell signaling finally contributing to the phenotypic plasticity that challenges the central dogma of molecular biology. This helps us in developing new treatments.

\section{Signaling Pathways}

In its watchful analysis of the pathways of tumor signaling, the Pan-Cancer Atlas discloses the patterns of vulnerabilities that serve as targetable weak links in the story of malignancy. Pathways studied include RAS (rat sarcoma), Ubiqutin, MYC, splicing, and DNA damage repair.
The Pan-Cancer Atlas can be likened to a "Google Earth" for human cancer research.

\section{Other Salient Clinical Outcomes}

a. Genes that are frequently mutated are not confined to one cancer type rather spread across different cancer types. An important observation that leads to the idea of "BASKET" trial in which different cancer types with a same targetable genetic alteration have been grouped into a basket and are being targeted with a single-drug type.

b. Data on mutation, gene expression, and methylation help us develop different subclassification of the existing cancer types and when analyzed in correlation with the clinical data have surprisingly shown us that they respond differently to treatments.

1. Important example of the above said is the story of low-grade gliomas. TCGA identified three subgroups of low-grade gliomas: isocitrate dehydrogenase (IDH) mutant with $1 \mathrm{p} / 19 \mathrm{q}$ codeletion, IDH mutant without 1p/19q codeletion, and IDH wild type. The IDH wild type shares similar genetic markers and clinical outcomes with glioblastomas, indicating that this variant of low-grade glioma may be a precursor to its more aggressive counterpart. This also embarked on the fact that they should not be treated like their other low-grade counterparts. ${ }^{2}$ A year after the above discovery, a new classification of brain tumors was put forth. The backbone of 2016 classification was the molecular signature that determined the risk grouping and the treatment strategy rather than the histopathology.

2. Another example is that of the uterine endometrial carcinoma. TCGA learned four different molecular subtypes of endometrial carcinoma, namely POLE (polymerase epsilon) ultra-mutated, microsatellite instability high, copy number low, and copy number high. This will soon be incorporated by the FIGO (Fédération Internationale de Gynécologie et d'Obstétrique) into the grading system $^{3}$ with more relevance to treatment and their differential response to chemotherapy, immunotherapy, and radiotherapy. The same is observed in "Molecular classification is prognostic of adjuvant treatment benefit in patients. This with high risk endometrial cancer $^{4 "}$ Creutzberg et al support the conclusions of TCGA therapeutically.

c. Newer targets with potential to challenge the present standard of care have been revealed. Some of them being cervical cancers with gene fusions involving Breast Cancer Anti-Estrogen Resistance 4 are sensitive to lapatinib. ${ }^{5}$

Bladder cancer was grouped into different molecular subtypes similar to endometrium that have different responses to the existing treatment. These subgroups elucidated by TCGA may show striking differences in the treatment chosen. The exiting features of the bladder cancer are patients with human epidermal growth factor receptor-2 protein 
expressions may respond to its directed treatments. Other important finding in the analysis of bladder cancer was the p53-dependent cell cycle alteration, which may benefit from counteracting the cyclin-dependent kinases with the CDK 4/6 group of drugs, namely palbociclib. PI3K/AKT pathway abnormalities might also lead to some new therapeutic targets.

Basal-like subtype of breast cancer shared many genetic features with that of high-grade serous ovarian cancer, suggesting that both of them might have a common molecular origin and may share many therapeutic opportunities.

And thus, the story goes on. but "A Journey of thousand miles begins with a single step." Looking forward to live the reality out of this dream.

\section{Financial Support and Sponsorship}

No financial aid was taken.

\section{Conflicts of Interest}

There are no conflicts of interest.

\section{References}

1 The Cancer Genome Atlas-Timeline and Milestones. National Cancer Institute; 2020. Available from: https://www.cancer. gov/about-nci/organization/ccg/research/structural-genomics/tcga/history/timeline. Accessed July 19, 2021.

2 Brat DJ, Verhaak RG, Aldape KD, et al; Cancer Genome Atlas Research Network. Comprehensive, integrative genomic analysis of diffuse lower-grade gliomas. $N$ Engl J Med 2015;372(26):2481-2498

3 Soslow RA, Tornos C, Park KJ, et al. Endometrial carcinoma diagnosis: Use of FIGO grading and genomic subcategories in clinical practice: recommendations of the international society of gynecological pathologists. Int J Gynecol Pathol 2019;38(Suppl 1):S64-S74

4 Creutzberg CL, Leon-Castillo A, De Boer SM, et al. LBA63 molecular classification of the PORTEC-3 trial for high-risk endometrial cancer: Impact on adjuvant therapy. Ann Oncol 2019;30(Suppl 5):mdz394-mdz060

5 Cancer Genome Atlas Research Network. Albert Einstein College of Medicine, Analytical Biological Services, Barretos Cancer Hospital, Baylor College of Medicine, Beckman Research Institute of City of Hope, et al Integrated genomic and molecular characterization of cervical cancer. Nature 2017;543:378-384 